\title{
AIP hamedodyes
}

\section{The role of coulomb interactions in valence transition: Falicov-Kimball model}

Samuel P. Bowen and S. C. Lady

Citation: Journal of Applied Physics 55, 1925 (1984); doi: 10.1063/1.333520

View online: http://dx.doi.org/10.1063/1.333520

View Table of Contents: http://scitation.aip.org/content/aip/journal/jap/55/6?ver=pdfcov

Published by the AIP Publishing

\section{Articles you may be interested in}

Ground state study of spin-1/2 Falicov-Kimball model on a triangular lattice

AIP Conf. Proc. 1447, 1133 (2012); 10.1063/1.4710407

Study of spinless Falicov-Kimball model on a square lattice away from half-filling

AIP Conf. Proc. 1447, 93 (2012); 10.1063/1.4709897

Thermodynamics of a spinless, extended Falicov-Kimball model on a triangular lattice AIP Conf. Proc. 1447, 89 (2012); 10.1063/1.4709895

Thermodynamics of the Two-dimensional Falicov-Kimball Model on the Triangular Lattice AIP Conf. Proc. 1349, 109 (2011); 10.1063/1.3605761

A Monte Carlo study of the Falicov-Kimball model in the perturbative regime Low Temp. Phys. 33, 797 (2007); 10.1063/1.2781508

MIT LINCOLN LABORATORY CAREERS

Discover the satisfaction of innovation and service to the nation
- Space Control

- Air \& Missile Defense

- Communications Systems $\&$ Cyber Security

- Intelligence, Surveillance and Reconnaissance Systems

- Advanced
Electronics
- Tactical Systems
- Homeland
Protection
- Air Traffic Control

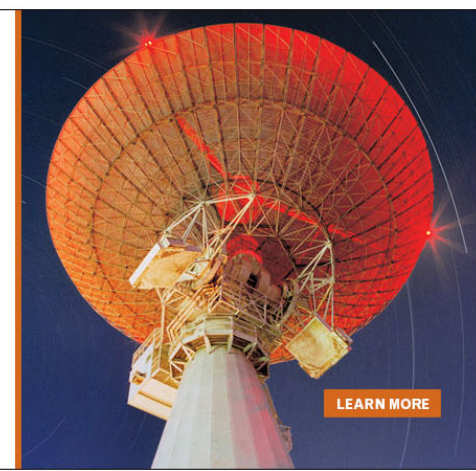




\title{
The role of coulomb interactions in valence transition: Falicov-Kimball model
}

\author{
Samuel P. Bowen and S. C. Lady \\ Department of Physics, Virginia Tech, Blacksburg, Virginia 24061
}

\begin{abstract}
A nonperturbative method of Green function calculation is applied to the Falicov Kimball model Hamiltonian. In an approximation to first order in the hopping matrix elements, self-consistent solutions for several thermal averages and correlation functions do not show abrupt phase changes as a function of temperature. This treatment suggests that the coulomb correlation by itself is not the key ingredient to understanding valence transitions.
\end{abstract}

PACS numbers: $71.45 . \mathrm{Gm}$

This paper presents a theoretical study of the Falicov Kimball (FK) model Hamiltonian ${ }^{1}$ as a first step in a larger program to apply a little used, but convergent Green function method ${ }^{2}$ to the study of valence fluctuations. The FK model has been chosen because of its simplicity both for use of the calculational method and as a test of the effect of the coulomb repulsion between $f$ and $d$ electrons at each site.

The form of the Hamiltonian used here is

$$
\begin{aligned}
H= & \sum_{l} E f_{l}^{+} f_{l}+\sum_{l} \epsilon d_{l}{ }^{+} d_{l} \\
& +g \sum_{l} f_{l}^{+} f_{l} d_{l}^{+} d_{l}+\sum_{l^{\prime}} t d_{l}{ }^{+} d_{l},
\end{aligned}
$$

the original form proposed by FK and first analyzed in a Hartree Fock (HF) approximation. ${ }^{3}$ Here $E$ and $\epsilon$ are the single-site atomic one particle energies of the $f$ orbitals and $d$ orbitals respectively, $g$ is the screened coulomb integral between the $f$ and $d$ orbitals on one site, and $t$ is the hopping matrix element for the $d$ orbitals. The $f$ orbitals are assumed to be so localized that they cannot hop from site to site. Note that because of this restriction the $N_{l}=f_{l}^{+} f_{l}$ is a constant of the motion for each $l$.

Even though there have been a large number of studies of more complicated versions of this model including spin, on-site hybridization, and static-site occupation fluctuations, this study is restricted to an examination of the simple no spin model (1). Besides demonstrating the methods which should have significant application on more realistic models, this model will allow study of the interplay of the hopping integrals $t$ and the coulomb integral $g$.

This study has merit since the earliest applications of this model, ${ }^{4}$ and even recent papers, ${ }^{5}$ study this model in the small $t$ limit and using Hartree approximations find a very rich phase diagram. The approximations and results to be shown here should have their greatest validity in the small $t$ regime, and are a step beyond the HF approximation, and will illustrate the well known fact that HF approximations over emphasize the tendency to phase changes. Our results will show a strong continuity with the atomic limit $(t=0)$ and smooth monotonic temperature dependence of a number of thermal correlation functions when $t \neq 0$.

The method we use to study this model has been discussed before, though several modifications have been recently discovered which will be discussed here and elsewhere. The method essentially introduces matrix techniques similar to those commonly used in band structure calcula- tions into the study of many-body physics. Combining a special set of stepping operators used intuitively by Hubbard ${ }^{6}$ and more generally elucidated by $B$. R. Judd ${ }^{7}$ with a convergence theorem by Masson, ${ }^{8}$ the method yields a sequence of approximations which are guaranteed to converge for any values of the model parameters. The main focus of this paper is one of the first approximants in the Masson sequence. This approximation seems to be quite rich in physics. It must be emphasized that this method removes the common ambiguity found in Zubarev ${ }^{9}$ decoupling schemes by noting that there is a further structure present among the stepping operators. This structure is that two second quantized operators $A$ and $B$ consisting of odd numbers of creation and annihilation generators can be considered as elements of a operator Hilbert space with a fermion (anticommutator) inner product

$$
[(B, A)]=\left\langle\left\{A, B^{+}\right\}\right\rangle
$$

where $(\ldots)$ denotes the thermal average in the grand canonical ensemble, and $\{. . . .$.$\} denotes the anticommutator. The$ starting point of the method is to note that the $L$ operator acting

$$
L_{\rho}=[\rho, k]
$$

on some arbitrary operator $\rho$ by commutation with the Hamiltonian $K=H-\mu N$, where $\mu$ is the chemical potential and $N$ is the total number operator, is a self-adjoint operator with respect to Eq. (2) and that the retarded anticommutator Green functions have time Fourier transforms which are matrix elements of the resolvent of $L$ in the Fermi space. Using the common Zubarev notation

$$
\left\langle\left\langle A ; B^{+}\right\rangle\right\rangle_{\omega}=\left\{\left[B,(\omega-L)^{-1} A\right]\right\},
$$

for possibly complex frequency $\omega$. The implication of this simple relationship is that a secular matrix for $L$ can be calculated algebraically with respect to an orthonormal sequence of operators some of which lie in the "plane" determined by $A$ and $B$. This secular matrix for $L$ will contain thermal averages of several different operators which must be determined as outlined below. Matrix elements of the resolvent Eq. (4) can be determined in the approximations simply by inverting the finite dimensional matrices of $\omega-L$. A surprising and powerful theorem by Masson states that the sequence of Green functions determined by calculating the inverse of ever larger secular matrices for $L$ converges to the exact Green function for any Hamiltonian as the size of the matrix increases. This paper will study only the first such 
approximant which will include all contributions to first order in $t$.

An important note regards the choice of basis operators that is used to calculate the secular matrices of $L$. The speed of convergence and relevance of the approximation in the thermodynamic limit are critically dependent on the choice of basis. In this study the first approximation will be studied in a generalized Bloch representation for many electron stepping operators. All of these ideas can be concretely realized by first briefly studying the atomic limit. Following Hubbard and Judd we can study all of the electron annihilation operators by studying the conditional many body annihilation operators for $r= \pm 1$.

$$
\begin{aligned}
& \psi_{l}^{(r)}=n_{l}^{(r)} f_{l}=\left[(1-r) / 2+r d_{l}^{+} d_{l}\right] f_{l}, \\
& \phi_{l}^{(r)}=N_{l}^{(r)} d_{l}=\left[(1-r) / 2+r f_{l}^{+} f_{l}\right] d_{l} .
\end{aligned}
$$

For $r=-1$ these operators correspond to an annihilation of an $f$ or $d$ electron at site $l$ if and only if the other electron is not present on the same site. The $r=+1$ operators corresponds to a change in particle number on site $l$ from 2 to 1 if and only if the other orbital is occupied. For this simple model these two events are the only ones so the following identities hold:

$$
\begin{aligned}
& f_{l}=\Psi_{l}^{(-)}+\Psi_{l}^{(+)}, \\
& d_{l}=\Phi_{l}^{(-)}+\Phi_{l}^{l+1} .
\end{aligned}
$$

The stepping operators $\Phi_{l}^{(r)}$ and $\Psi_{l}^{(r)}$ are eigenvectors of $L$ with eigenvalues $E^{(r)}=E+g(1+r) / 2 \quad$ and $\epsilon^{(r)}=\epsilon+g(1+r) / 2$. For the atomic limit $(t=0)$ these eigenvectors simplify the calculation of Green functions and equations for determining thermal averages of $n=\left\langle d_{l}{ }^{+} d_{l}\right\rangle$ and $N=\left\langle f_{i}^{+} f_{l}\right\rangle$ reduce to a simple linear set

$$
\begin{aligned}
& N=(1-n) f(E-)+n f(E+), \\
& n=(1-N) f(\epsilon-)+N f(\epsilon+),
\end{aligned}
$$

where $f\left(E^{(r)}\right)=\left[1+\exp \beta\left(E^{(r)}-\mu\right)\right]^{-1}$ is the Fermi function.

The solution of Eqs. (7) yield very smooth functions for $N$ and $n$ as temperature varies. As is well known, but not clearly documented in the literature, these results are in direct contradiction with the original HF approximation of Falicov and Kimball when $t=0$.

The study of the $t \neq 0$ case is more complicated since neither $\Phi_{l}^{(r)}$ nor $\Psi_{l}^{(r)}$ are eigenvectors of the $L$ using the full Hamiltonian. This obviously arises from the additional physical possibility for annihilating a $d$ electron at the $l$ th site by allowing it to jump to a neighboring site at which another $d$ electron has been annihilated. The details of our truncation must be discussed elsewhere due to space limitations, but the basic outline can be given below. We truncate the $L$ matrix using a Gramm-Schmidt procedure and include only the following generalized Bloch state operators:

$$
\begin{aligned}
& \phi_{k}^{(r)}=\frac{1}{\sqrt{N_{0}}} \sum_{l} e^{i k \cdot R_{l}} \phi_{l}^{(r)}, \\
& \psi_{k}^{(r)}=\frac{1}{\sqrt{N_{0}}} \sum_{l} e^{i k \cdot R_{l}} \psi_{l}^{(r)} .
\end{aligned}
$$

With respect to these basis states the secular matrix for $L$ reduces to $4 \times 4$ consisting of two independent $2 \times 2$ blocks. For $r=-1$ these equations reduce to

$$
\begin{aligned}
L \phi_{k}^{(r)}= & \left.\left(\epsilon^{(r)}+t S(k)\left\langle N_{l}^{(r)} N_{l}^{(r)}\right\rangle / N^{(r)}\right)\right) \phi_{k}^{(r)} \\
& +\frac{t S(k)\left\langle N_{l}^{(r)} N_{l}^{(-r)}\right\rangle}{\sqrt{N(1-\bar{N}-\bar{r}} \phi_{k}^{(r)},} \\
L \psi_{k}^{(r)=} & \left(E^{(r)}+\frac{z t \bar{\lambda}}{\left\langle n^{(r)}\right\rangle}\right) \psi_{k}^{(r)}-\frac{z t \tilde{\lambda}}{\sqrt{n(1-n)}} \psi_{k}^{(-r)},
\end{aligned}
$$

where $S(k)$ is the usual nearest neighbor structure factor for the tight binding band, $\left\langle N_{l} N_{l}\right\rangle$ is a two site correlation function where $l$ and $l^{\prime}$ denote nearest neighbor sites, $z$ is the number of nearest neighbors and

$$
\tilde{\lambda}=\left\langle\left(\phi_{l}^{++1}\right)^{+} d_{l},-d_{l}{ }^{+} \phi_{l}^{!-1}\right\rangle
$$

is the nearest-neighbor correlation function which measures the $d$-electron correlation on the near-neighbor sites. These equations indicate two general features which one must expect from band structures based on such strongly correlated Bloch states. First, many of the band properties will exhibit a temperature dependence not to be found in the usual $\mathbf{H F}$ band theories. As seen above the hopping matrix elements and the corresponding subband width are modulated by thermal factors which reflect the occupation and correlation between sites in the lattice. Also present is the $\tilde{\lambda}$ coefficient which is essentially a temperature dependent chemical shift for each site.

All of these thermal averages can be calculated within the Fermi space using the standard detailed balance condition of anticommutator Green functions with the exception of the two site correlation function $\left\langle N_{l} N_{l}\right\rangle$. To calculate this two site thermal average a method very similar to that outlined above for anticommutator Green functions has been developed for two site commutator Green functions. ${ }^{10}$ This development uses another operator Hilbert space, called the Bose space with a different inner product, ${ }^{11}$ the same super operator $L$, and corresponding detailed balance conditions. The simplest approximant making one by one truncations of the two-electron stepping operators yields a set of closed equations for two site thermal averages containing two, three and four factors of $N$ and/or $n$ for each site.

These equations in conjunction with the Bloch state analogues of Eq. (7) comprise a set of eight equations which can be solved for any temperature and chemical potential for the relevant thermal averages. At high and low temperatures and chemical potentials these systems have analytic solutions, but at intermediate values they must be solved numerically. With a chemical potential chosen to yield one particle per site, the temperature dependence of $n, N$ and $\left\langle N_{l} N_{l}^{\prime}\right\rangle$ as well as the other correlation functions is quite smooth and devoid of the abrupt phase transitions seen by other approximation techniques. For small $t$ where an approximation is essentially exact the results evolve smoothly from the atomic limit results. This smooth behavior suggests that the coulomb repulsion $g$ in this simple model is not, by itself, the key physical effect inducing metal-insulator, valence transitions as a function of temperature.

The techniques described here constitute a potentially 
powerful method for studying strongly correlated band structures and systems with strong fluctuations between different configurations. The method is being applied to other models and will be more completely discussed elsewhere.

'L. M. Falikov and J. Kimball, Phys. Rev. Lett. 22, 997 (1969).

${ }^{2}$ S. P. Bowen, J. Math. Phys. (NY) 16, 420 (1975); Phys. Rev. B 18, 3400 (1978).

${ }^{3}$ S. P. Bowen, J. Math. Phys. (NY) 16, 420 (1975); Phys. Rev. B 18, 3400 (1978); R. Ramirez, L. M. Falicov, and J. C. Kimball, Phys. Rev. B 2, 3383 (1970); P. Schlottman and L. M. Falicov, Phys. Rev. B 23, 5916 (1981).
${ }^{4}$ S. P. Bowen, J. Math. Phys. (NY) 16, 420 (1975); Phys. Rev. B 18, 3400 (1978).

${ }^{5}$ M. E. Foglio and L. M. Falicov, Phys. Rev. B 20, 4554 (1979), M. E. Foglio, C. A. Balseiro, and L. M. Falicov, Phys. Rev. B 20, 4560 (1980); M. E. Foglio and L. M. Falicov, Phys. Rev. B 21, 4154 (1980).

${ }^{6} \mathrm{~J}$. Hubbard, Proc. R. Soc. (London) Ser. A 176, 238 (1963).

${ }^{7}$ B. R. Judd, "Topics in Atomic Theory," Topics in Atomic and Nuclear Theory (University of Canterbury, New Zealand, 1970), p. 21-25.

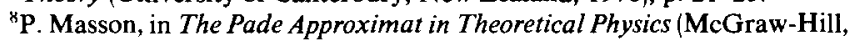
New York, 1971).

${ }^{9}$ D. Zubarev, Usp. Fiz. Nauk 71, 71 (1960).

${ }^{10}$ Samuel P. Bowen, C. D. Williams, and S. C. Lady (to be published).

${ }^{11}$ S. P. Bowen, J. Math. Phys. (NY) 16, 420 (1975); Phys. Rev. B 18, 3400 (1978). 